\title{
Rilievo digitale e analisi dell'ala lusignana del castello di Kyrenia
}

\author{
Digital survey and analysis of the lusignan wing of Kyrenia Castle
}

\author{
Matteo Bigongiari ${ }^{\text {a }}$, Federico Cioli ${ }^{\text {b }}$ \\ Dipartimento di Architettura - Università degli Studi di Firenze, Florence, Italy \\ a matteo.bigongiari@unifi.it; ${ }^{\mathrm{b}}$ federico.cioli@unifi.it
}

\begin{abstract}
The paper illustrates the documentation and analysis methods of a wing of the Kyrenia castle in Cyprus. The laser-scanner and photogrammetry Structure from Motion (SfM) survey campaigns took place during the international workshop "Reading and designing the Kyrenia Castle" held in May 2018 in Girne, involving the Girne American University (Cyprus) and the University of Florence. The survey required a more in-depth analysis of the environments around the courtyard, in particular those located on the eastern front, which host numerous archaeological finds, including the Kyrenia shipwreck, dating back to the fourth century BC. The analysis was based on the drawings (plans, elevations and sections) served as support for diagnostic and material investigations aimed at highlighting the state of superficial conservation and structures and their historical stratigraphy.
\end{abstract}

Keywords: Kyrenia castle, digital survey, diagnostic analysis, conservation.

\section{Cenni storici e architettonici sul castello di Kyrenia nel periodo lusignano ${ }^{1}$}

La storia di Cipro è caratterizzata dalla forte influenza di numerose culture, rappresentando un territorio di confine tra Asia, Africa ed Europa. L'isola infatti venne colonizzata dai Greci micenei, dai califfati arabi per un breve periodo, dalla casata francese dei Lusignano, dai Veneziani ed in seguito dagli Ottomani tra il 1571 e il 1878 (Mirbagheri, 2009). Questa varietà culturale si riflette nelle architetture dell'isola e il Castello di Kyrenia a Girne ne rappresenta una sintesi esemplare.

Il Castello di Kyrenia è situato in un sito che testimonia tracce di avamposti fortificati sin dalla preistoria e segni del periodo ellenistico e romano (111-11 a.C.). Le fasi evolutive del castello, situato ad est del porto, si possono suddividere in tre principali periodi storici: il periodo bizantino (VII-XII secolo d.C.), il periodo lusignano (1208-1211 d.C.) e il periodo veneziano (1489-
1570 d.C.). Una prima descrizione storica del castello è reperibile nei "Viaggi" di W. De Oldenburg, che visitò Cipro nel 1211 sotto il regno di Re Ugo I, descrivendo Girne come "una cittadina ben fortificata, che ha un castello con mura e torri, il cui vanto principale è un buon porto"2. Dalla descrizione di un viaggiatore medievale del XIV secolo il castello appariva come un edificio di pianta quadrata con quattro torri angolari e provvisto di un porto interno che venne successivamente trasformato in fossato dagli ingegneri veneziani. Ospitava inoltre gli appartamenti reali, che nel XVI secolo, con la trasformazione del castello ad opera dei veneziani, vennero convertiti in celle di prigionia nella parte settentrionale, mentre a sud vennero riempite di terra per rafforzare le mura difensive (Jeffrey, 1918). Nel corso della sua storia il castello di Kyrenia ha subito numerosi assedi che si sono quasi 
sempre risolti tramite armistizi o cessioni, le variazioni della sua immagine sono infatti principalmente dovute non a distruzioni, bensì all'adeguamento architettonico e strutturale all'evolversi delle tecnologie belliche. Tra il 1229 e il 1232, il castello fu posto sotto assedio sotto la guida di Filippo di Novara, con l'uso di torri di legno e catapulte, e venne ceduto dagli imperialisti nel 1233 (Jeffrey, 1918). Un altro grande assedio avvenne nel 1374 da parte delle forze genovesi e durò più di un anno, portando alla distruzione di buona parte della fortificazione. Si concluse con la consegna del castello nelle mani degli invasori da parte del conestabile, futuro re Giacomo I, e con esso l'intera isola divenne un possedimento tributario delle Repubbliche italiane. Con l'invasione dei mamelucchi nel 1426, Kyrenia divenne una delle principali roccaforti del governo. Il cardinale Ugo di Lusignano, fratello del re Giano, raccolse i tesori e i membri della famiglia reale e si rifugiò nella fortezza settentrionale. Il resto dell'isola cadde in balia dei saccheggi e delle stragi da parte dei mamelucchi e i servi, rinunciando ai loro obblighi feudali, istituirono una sorta di repubblica libera. Con il ritorno del re, il 15 marzo 1427 vennero ristabiliti la legge e l'ordine e Girne fu designato come luogo di sbarco per i festeggiamenti reali (Jeffrey, 1918). Nel 1460 il castello divenne il rifugio della legittima Regina di Cipro Carlotta di Lusignano, figlia del Re Giovanni II, posta sotto assedio per circa tre anni dal fratellastro usurpatore Giacomo II. La guerra civile portò alla disfatta del regno lusignano e Cipro passò sotto l'occupazione veneziana.

Durante il periodo Lusignano l'aspetto militare del castello venne fortemente incrementato attraverso la costruzione di un nuovo ingresso, di una piazza, di torri a ferro di cavallo, di feritoie per arcieri e dei sotterranei. La difficoltà di ricostruire l'immagine del castello durante il periodo lusignano risiede nel fatto che una buona parte delle mura medioevali non sono più visibili a causa dell'ampliamento, dell'assedio e della ricostruzione ad opera dei veneziani motivata dall'introduzione della polvere da sparo.

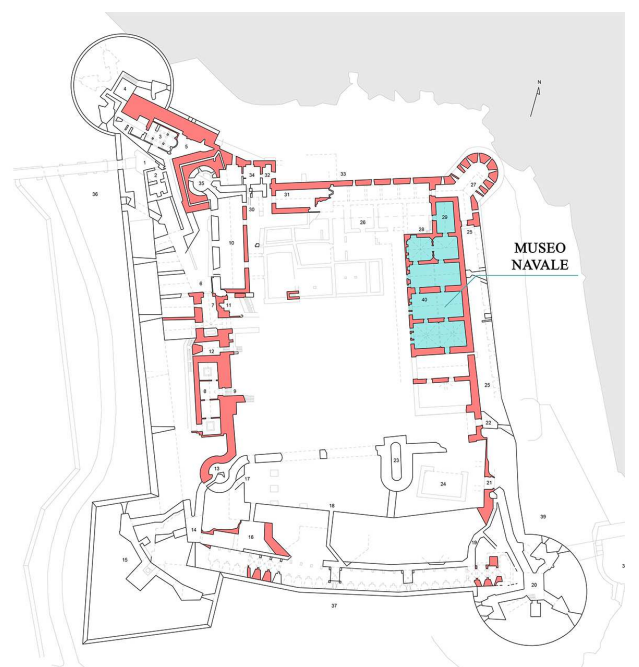

Fig. 1. Planimetria del castello con individuazione della fase lusignana e dell'area adibita a museo navale.

Il castello non venne costruito per difendere $\mathrm{Ky}-$ renia dagli attacchi dal mare, bensì per proteggere il porto dagli attacchi da terra e garantire l'approvvigionamento delle merci (Newman, 1947). Così come appariva durante il periodo lusignano, il castello di Kyrenia era costituito da una corte centrale quadrata circondata da mura merlate con torri circolari ad ogni angolo. I lati esposti ad est e a nord erano protetti dal mare, motivo per il quale presentano ancora una porzione delle mura lusignane. I veneziani infatti non rafforzarono questi due lati della fortezza. Il lato verso ovest, diretto verso il porto, era quello di ingresso al castello. Il lato sud era quello maggiormente esposto agli assedi, probabile motivo per il quale è quello che mostra meno segni del periodo lusignano. L'immagine attuale della fortezza è dovuta principalmente alla ristrutturazione e all'ampliamento intrapresi dai veneziani dal 1540 al 1544 , che ha visto la sostituzione delle torri quadrate con torrioni circolari, più resistenti ai colpi di cannone, e l'apertura di bocche per i cannoni sul versante verso terra, per timore di un attacco da parte degli Ottomani. Il castello però non venne mai attaccato, nel 1570 venne ceduto agli Ottomani, che inviarono ai Veneziani le teste dei loro comandanti a Nicosia, e lo presidiarono per circa tre secoli (Camiz, et al., 2016). Verso la fine dell'Ottocento 
l'amministrazione coloniale britannica lo utilizzò come carcere e scuola di polizia, realizzando alcune opere di restauro, come il rifacimento della cupola della piccola chiesetta di San Giorgio, inglobata all'interno delle mura veneziane (Volzone, et al., 2019).

Attualmente il castello è visitabile e ospita al suo interno un museo, collocato nel lato est del cortile centrale, all'interno del quale sono raccolti numerosi reperti archeologici dell'antica Grecia e il relitto della Nave di Kyrenia, una nave mercantile del IV secolo a.C. recuperata sulle sponde cipriote nel 1967. Il museo si struttura all'interno di una grande costruzione del periodo lusignano posta nel lato nord-est del cortile, a ridosso delle mura. Il contributo nello specifico riguarda il rilievo e l'analisi dello stato di conservazione dell'ala Lusignana del castello di Kyrenia. La ricerca è iniziata attraverso un seminario e un workshop dal titolo Reading and Designing the Kyrenia Castle. Enhancing heritage in the historical landscape, che si sono tenuti presso la Facoltà di Architettura della Girne American University di Cipro tra il 6 e il 13 maggio $2018^{3}$. Il workshop ha coinvolto cinquantasei studenti delle tre università, che sono stati divisi in gruppi al fine di coprire gli argomenti di progettazione e rilievo digitale sotto la guida dei loro tutor (Camiz, et al., 2018). Le fasi di documentazione hanno visto l'interazione di varie professionalità negli ambiti del rilievo, della storia, dell'architettura, dell' archeologia e della progettazione e hanno portato alla realizzazione di campagne di rilievo laser-scanner e fotografiche Structure from Motion che hanno interessato l'intera fortezza e le sue componenti. L'indagine di seguito descritta ha richiesto un'analisi più approfondita degli ambienti intorno al cortile, in particolare quelli situati sul fronte orientale, che ospitano numerosi reperti archeologici, tra cui il relitto di Kyrenia, risalente al IV secolo a.C. L'analisi si basa sui disegni (piani, prospetti e sezioni) serviti da supporto per indagini diagnostiche e materiche volte ad evidenziare lo stato di conservazione superficiale e strutturale degli apparati murari e la loro stratigrafia storica. In seguito alle fasi di rilievo ed analisi sul campo, il lavoro è proseguito all'interno di un seminario tematico dell'DIDA che ha previsto l'interazione di quattro discipline: il rilievo, il restauro, la progettazione e la comunicazione, sotto la supervisione dei proff. Stefano Bertocci, Giovanni Minutoli, Andrea Ricci e Paola Puma. Il seminario si è concentrato sull'ala del Museo Archeologico all'interno del Castello, che richiede un nuovo schema di distribuzione per le sale interne, attraverso un'indagine dei sistemi costruttivi, delle principali problematiche di conservazione e delle condizioni strutturali dei vani ospitanti il museo navale.

\section{Il rilievo digitale}

Il rilievo digitale dell'ala museale del castello di Kyrenia è stato impostato con il proposito di utilizzare gli elaborati grafici tradizionali, ovvero piante sezioni e prospetti, come basi morfologiche, come contenitori pronti ad ospitare le mappe tematiche utili all'analisi strutturale dell'edificio. Ai fini dell'acquisizione delle misurazioni quindi il rilievo è stato progettato per garantire la descrizione di tutte le superfici pertinenti l'ala analizzata con un dettaglio adeguato a quello previsto dalla scala di restituzione necessaria a supportare tali indagini, ovvero almeno 1:50. L'edificio è stato interessato, come tutto il resto del complesso, da una campagna di acquisizione di scansioni laser scanner (è stato utilizzato uno strumento a differenza di fase $\mathrm{Z}+\mathrm{F}$ Imager 5006H) con la necessità di garantire una densità della maglia di punti sulle superfici adatta alla scala di dettaglio, con una distanza tra i punti rilevati inferiore ad $1 \mathrm{~cm}$. Per verificare che il modello di punti avesse un errore inferiore a quello ammissibile dalla scala di restituzione prescelta, ovvero $1 \mathrm{~cm}$ per la scala 1:50, dopo aver eseguito una prima fase di registrazione delle scansioni mediante sovrapposizione di punti ${ }^{4}$, è stato necessario eseguire sul modello di punti finale una fase di collaudo delle rototraslazioni che ne garantisse l'affidabilità: il modello è stato sezionato da piani orizzontali e verticali per indagare che il disallineamento dei fili di sezione delle nuvole di punti ${ }^{5}$ fosse inferiore al centimetro desiderato. La nuvola di punti è stata successivamente elaborata per realizzare i piani di sezioni utili a descrivere tutte le sezioni necessarie a descrivere le murature dell'edificio. 

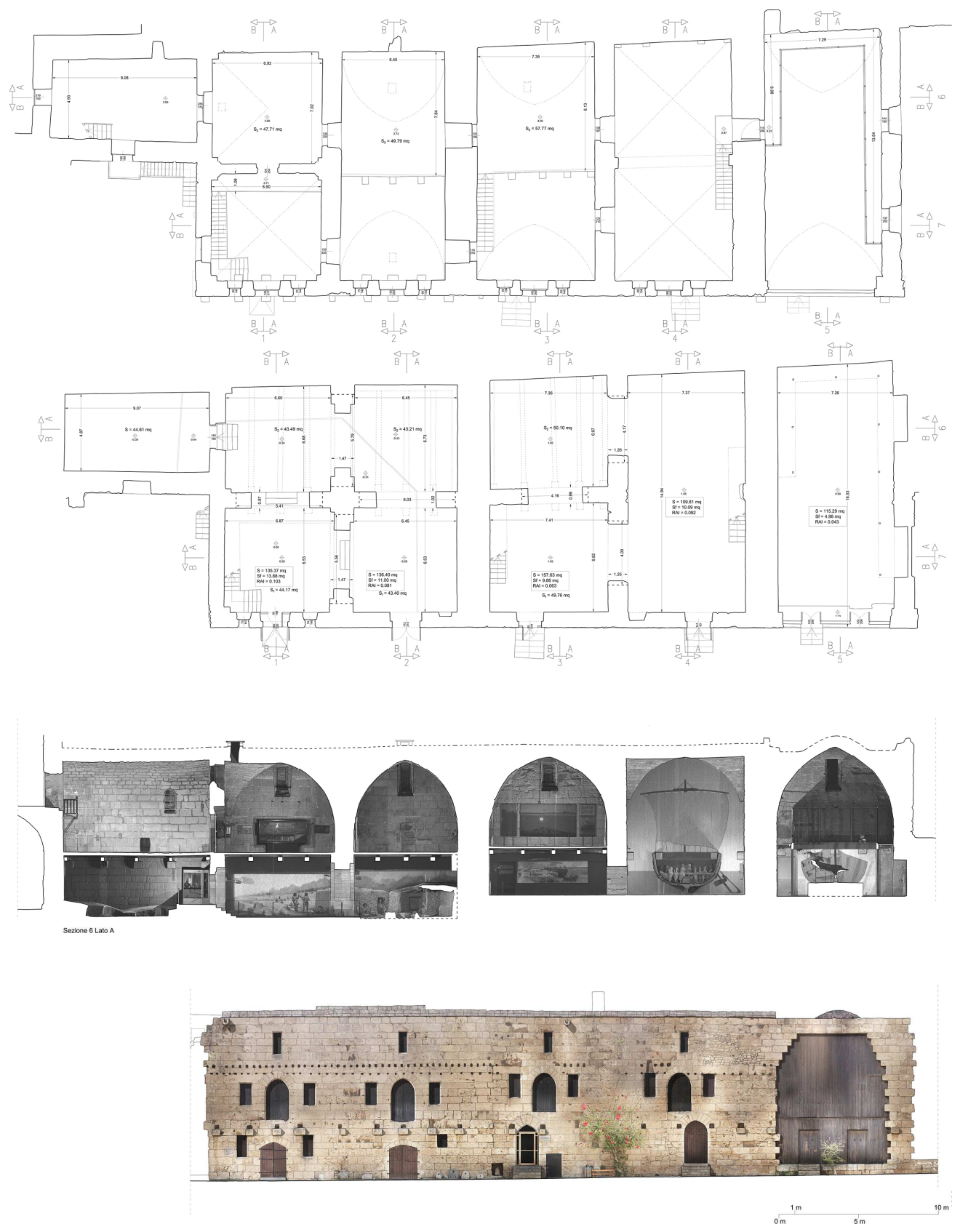

Fig. 2. Piante, sezioni e prospetto esterno con fotopiano dell'ala lusignana del Castello di Kyrenia adibita a museo navale. 
Le esportazioni di immagini rettificate ad alta risoluzione ha consentito restituire su piattaforme di disegno CAD, di restituire il rilievo a filo di ferro in modo da descrivere la tessitura muraria di ogni superficie.

Il rilievo morfologico è stato infine integrato con il dato colore grazie alla realizzazione di accurati fotopiani, calibrati sulla nuvola di punti ${ }^{6}$, ottenuti attraverso procedure Structure from Motion che hanno consentito di realizzare modelli tridimensionali di tutti gli ambienti esterni ed interni dell'edificio e di estrarne la texture utile a descrivere la matericità di tutte le superfici analizzate.

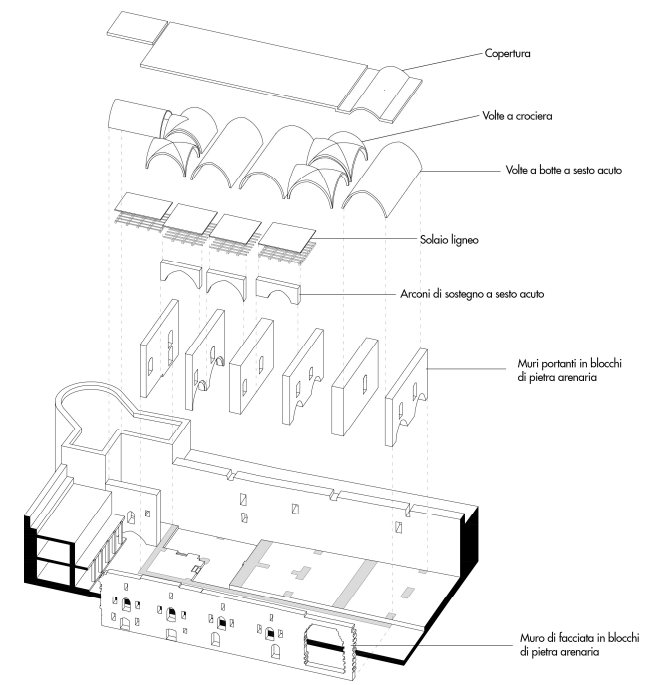

Fig. 3. Esploso assonometrico degli ambienti interni e degli elementi strutturali dell'ala museale.

\section{Le analisi}

I risultati del rilievo digitale dell'edificio analizzato hanno consentito di sviluppare le analisi strutturali, in modo tale da perseguire quel percorso di conoscenza, intrapreso con lo studio delle fonti storiche e approfondito con un adeguato rilievo morfologico del fabbricato, che consenta di determinare in modo scientifico lo stato di conservazione delle murature (Minutoli, 2017). L'accuratezza con cui sono state disegnate le strutture voltate che compongono l'edificio, ha permesso in primo luogo di vedere in maniera speditiva se il profilo delle murature seguisse un andamento regolare, oppure se fosse invece in alcuni punti deformato. Per comprendere al meglio il comportamento statico dell'edificio è stato necessario realizzare un modello schematico che ne illustrasse le strutture: le cinque gallerie che costituiscono l'ala della fortificazione, sono tutte voltate in pietra, alcune a botte con sesto acuto, altre a crociera, incrociando comunque due profili acuti; non seguono un andamento ortogonale al muro di cinta esterno della fortificazione, che era stato realizzato in una fase precedente, e appaiono in numero inferiore rispetto all'originario edificio che si estendeva almeno per due ulteriori campate come è ben visibile dalle tracce delle strutture voltati presenti oltre la campata che ospita il museo del relitto navale.

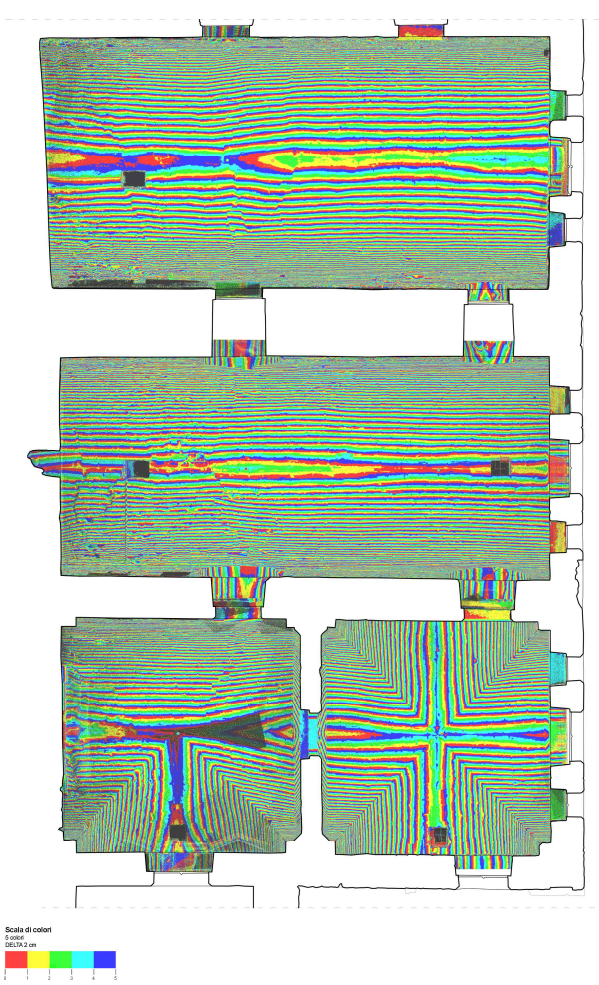

Fig. 4. Elevation Map dei sistemi voltati.

Da una prima osservazione è evidente che l'alternanza di tipologia di volta sia dovuto ad un importante intervento di restauro, in seguito a forti dissesti delle strutture che ha portato a sostituire le originarie volte a botte con le crociere: l'evidenza di questi interventi è dovuta alla tota- 
le assenza di lesioni sulle murature voltate a crociera, invece numerose nelle volte a botte e nelle murature portanti sottostanti che hanno causato forti dissesti come testimoniano ad esempio le architravi fortemente dissestate delle aperture tra i vani. Da una analisi del fronte è probabile che l'edificio avesse almeno una ulteriore campata verso la torre angolare, motivo che potrebbe spiegare la scelta di modificare il profilo delle precedenti volte a botte, che non può essere pratico, data la maggiore complessità del realizzare una crociera, ma statico, per evitare di fornire una spinta uniforme su tutto un muro ormai privo della contro-spinta della volta contigua. Per verificare lo stato di conservazione geometrica delle volte e delle murature portanti che le sorreggono è stata svolta una puntale analisi delle mappe di deformazione (elevation map) estratte direttamente dalle nuvole di punti; i fuori piombo delle murature sono stati estratti con la metodologia tradizionale che da diversi anni ormai viene utilizzata all'interno del gruppo di ricerca ${ }^{7}$. Dato che era possibile supporre che nonostante grandi dissesti delle volte, le spesse murature di una fortificazione non mostrassero gravi segni di spostamento è stato cercato un metodo di investigazione del profilo delle volte: utilizzando le mappe di deformazione come se fossero curve di livello è stato possibile indagare se le curvature delle strutture voltate seguissero infatti una geometria regolare ${ }^{8}$, notando come non siano presenti accentuate irregolarità di forma eccezion fatta per i punti fortemente interessati da antiche lesioni strutturali ormai risarcite. Con molta probabilità il dissesto che era presente nelle volte è stato ripristinato quando è stata realizzata la nuova copertura piana protetta da uno strato di guaina impermeabile, a cui si accede mediante il camminamento lungo le cortine murarie.

Non è possibile purtroppo comprendere più il reale sviluppo volumetrico che questo edificio presentava anche a causa della perdita di tutte quelle strutture leggere, lignee, che dovevano consentire la distribuzione dall'esterno all'interno dei locali, mentre è sempre ben leggibile dal prospetto dell'edificio la collocazione dei travetti lignei che insieme agli impalcati verticali, di cui si possono osservare alcune tracce, o meglio i segni degli alloggi delle travi, nella pavimentazione di fronte all'edificio, sostenevano i tavolati lignei; dall'analisi delle stratigrafie murarie $^{9}$ si osserva che mentre nelle prime due campate dall'angolo della fortificazione le tracce di queste strutture sono regolari e coerenti con l'altezza delle porte, dalla terza campata le porte e le finestre del secondo piano sono rialzate mentre le tracce delle travi rimangono ad altezza invariata, cosa che fa intuire ad un certo punto ad una modifica del progetto delle aperture, coerente con il rialzamento interno della pavimentazione delle tre campate seguenti; allo stesso modo cambia la tessitura muraria e le dimensioni dei blocchi di arenaria, che divengono di misura ridotta, e sono notevoli le modifiche alla tessitura, di piccola taglia e irregolare, che si trovano a volte intorno alle porte, fortemente rialzate, a cui si accede tramite scale esterne. Lo studio delle deformazioni della facciata ha evidenziato una ottima regolarità costruttiva: è comunque evidente come la sala del museo del relitto sia stata ritagliata in una campata su cui è stata smembrata la facciata esterna, probabilmente ai fini della conservazione della nave ritrovata.

Anche l'interno dell'edificio, adattato oggi a museo, non riesce a restituire l'immagine che un tempo dovevano avere queste gallerie, che sono oggi divise in due campate, quella addossata al muro perimetrale è soppalcata e ospita un piano superiore; i solai lignei alloggiano su mensole disposte su un muro ortogonale alle gallerie aperto da un arco a sesto acuto che permette il passaggio al piano terreno: questi archi si trovano anche aperti lateralmente, nelle murature che portano le volte, consentendo il passaggio tra una e l'altra galleria; al piano superiore invece le aperture nella prima campata di ciascuna volta fanno supporre che in realtà, come nella maggior parte delle gallerie delle fortezze crociate, ogni ambiente fosse completamente soppalcato in legno e fosse collegato dal piano superiore attraverso modeste aperture lateralmente agli altri ambienti: la stessa cosa accade con l'ambiente di collegamento alla torre lusignana, con cui l'edificio era collegato attraverso aperture laterali, e che doveva aprirsi al pian terreno sul cortile con un ampio arco acuto come le altre campate. 


\section{Conclusioni}

La conoscenza del complesso del castello di Kyrenia, grazie all'utilizzo del rilievo digitale, è stata notevolmente approfondita; lo studio delle fonti storiche, unito ad un rilievo morfologico affidabile, hanno consentito di sviluppare ulteriori analisi sullo stato di conservazione e sull'evoluzione dell'ala lusignana, che oggi svolge la funzione di sale espositive, integrando al suo interno le sale dell'antico relitto greco: in seguito alle indagini deformative e allo studio dei dissesti strutturali è stato possibile comprendere le problematiche che hanno danneggiato l'edificio nel passato e verificarne con metodi speditivi la stabilità statica di tutte le sue strutture. Un approfondito rilievo morfologico, integrato del dato fotografico ha inoltre reso possibile approfondire l'evoluzione del fabbricato in relazione alle stratigrafie presenti sulle murature a vista.

\section{Note}

${ }^{1}$ Il paragrafo 1 è stato scritto da F. Cioli, i paragrafi 2, 3 e 4 sono stati scritti da M. Bigongiari.

2 "A small town well fortified, which has a castle with walls and towers, its chief boast is a good harbor" (Jeffrey, 1918, p. 308).

${ }^{3}$ Il worksop e il seminario sono stati organizzati dalla Girne American University, Faculty of Architecture, Design and Fine Arts, (Prof. Alessandro Camiz) e dall'International Centre for Heritage Studies, Girne American University Girne, North Cyprus. È stato svolto in collaborazione con la Özyeğin University, Faculty of Architecture and Design, Department of Interior
Architecture and Environmental Design, Istanbul prof.ssa Zeynep Ceylanlı), il Department of Antiquities and Museums, TRNC, il Dipartimento di Architettura (DIDA), Università di Firenze (Proff. Stefano Bertocci e Giorgio Verdiani), la Scuola di Specializzazione in Beni Architettonici e del Paesaggio e il Dipartimento di Storia, Rappresentazione e Restauro dell'Architettura dell'Università Sapienza di Roma.

${ }^{4}$ Per una più approfondita discussione sulle tecniche di allineamento per sovrapposizione di porzioni di nuvole di punti vedi Bigongiari, 2017.

${ }^{5}$ La certificazione del rilievo è fondamentale ai fini della affidabilità delle restituzioni, cfr. Pancani, 2017.

${ }^{6}$ La nuvola di punti è servita come base morfologica per estrarre le misure e quindi la scala dei modelli, inoltre l'affidabilità metrica della texture estratta è stata verificata sovrapponendola all'immagine ad alta risoluzione della nuvola di punti, cfr. Pancani, 2017b.

${ }^{7}$ L'evoluzione dello studio delle deformazioni tramite l'utilizzo delle nuvole di punti può essere visto in Bertocci, 2015; Bertocci, et al., 2015.

${ }^{8}$ Se le curve di livello si spostano seguendo un profilo regolare e coerente con la direzione di sviluppo della volta è ipotizzabile che sia priva di deformazioni.

${ }^{9}$ Lo studio delle stratigrafie è indispensabile a comprendere l'evoluzione di un edificio e le sue ricadute sul modello statico originale Arrighetti, et al., etc.

\section{Bibliography}

Arrighetti, A.; Minutoli, G. (2018). "A multidisciplinary approach to document and analyze seismic protection techniques in Mugello from the Middle Ages to Early Modern Time”, Annals of Geophysics, 61.

Bertocci, S. (2015). "Il contributo del rilievo urbano dei centri storici italiani per il recupero e la prevenzione della vulnerabilità sismica: alcuni casi studi in Abruzzo, Toscana ed Emilia”, in Marotta, A.; Novello, G., Disegno e città. Drawing and city. Cultura arte scienze informazione, Gangemi, Roma.

Bertocci, S.; Minutoli, G.; Pancani, G. (2015). "Rilievo tridimensionale e analisi dei dissesti della Pieve di Romena", Disegnare con., 8-14. 
Bertocci, S.; Verdiani, G.; Sevketoglu, M. (2019). "Graffiti photogrammetry, extracting the signs from the walls of the Kyrenia Castle", in Proceedings of the CHNT/VH Conference, Museen der Stadt Wien - Stadtarchäologie, Vienna.

Bigongiari, M. (2017). "Il rilievo del centro storico di Brolo", in Arrighetti, A.; Gentile, S.; Minutoli, G., Bianca terra, Brolo, Armenio.

Camiz, A.; Bercigli, M. (2019). "UAV photogrammetry of large fortifications with low-cost tools a Cyprus experience", in Proceedings of the CHNT/VH Conference, Museen der Stadt Wien - Stadtarchäologie, Vienna.

Camiz, A.; Khalil, S.; Cansu Demir, S.; Nafa, H. (2016). "The Venetian defense of the Mediterranean: the Kyrenia Castle, Cyprus (1540-1544)", in Verdiani, G. ed., FORTMED 2016. Defensive architecture of the Mediterranean. XV to XVIII Centuries, DIDApress, Firenze, vol. 3, pp. 371-378.

Camiz, A.; Verdiani, G.; Ceylanli, Z. (2018). "International workshop: reading and designing the Kyrenia castle", in Bertocci, S. ed., Conference Proceedings of Symposium of representation scientific area for development of multidisciplinary international programs, DIDApress, Florence, pp. 264-298.

Ceylanlı, Z.; Özen, P.; Çiçek, E.; Arslan, P. (2019). "Evolving from castle to virtual space: the case of Kyrenia Shipwreck Museum", in Proceedings of the CHNT/VH Conference, Museen der Stadt Wien - Stadtarchäologie, Vienna.

Jeffery, G. (1918). A description of the Historic Monuments of Cyprus, William James Archer, Nicosia.

Minutoli, G. (2017). Percorsi di conoscenza per la salvaguardia della città storica, DIDApress, Firenze.

Mirbagheri, F. (2009). Historical Dictionary of Cyprus, Scarecrow Press.

Newman, P. (1970). The fortress of Kyrenia in the thirteenth century, during the Lusignan dynasty in Cyprus: Reconstructed from the existing remains, K. Rustem \& Brother, Nicosia.

Pancani, G. (2017a). La Città dei Guidi: Poppi. Il costruito del centro storico, rilievi e indagini diagnos-tiche, Edifir, Firenze.

Pancani, G. (2017b). "Rilievo delle lastre tombali del Camposanto Monumentale di Piazza dei Miracoli a Pisa", Restauro Archeologico, 2.

Volzone, R.; Bigongiari, M.; Cioli, F. (2019). "The church of St. George in the Kyrenia Castle in North Cyprus, bringing out the shape of architecture", in Proceedings of the CHNT/VH Conference, Museen der Stadt Wien Stadtarchäologie, Vienna. 